\title{
Nonreciprocal linear dichroism observed in electron spin resonance spectra of the magnetoelectric multiferroic $\mathrm{Pb}(\mathrm{TiO}) \mathrm{Cu}_{4}\left(\mathrm{PO}_{4}\right)_{4}$
}

\author{
Mitsuru Akaki $\odot,{ }^{1,2, *}$ Kenta Kimura $\odot,{ }^{3}$ Yasuyuki Kato $\odot,{ }^{4}$ Yuya Sawada, ${ }^{2}$ Yasuo Narumi, ${ }^{2}$ Hitoshi Ohta, ${ }^{1}$ \\ Tsuyoshi Kimura $\odot,{ }^{3}$ Yukitoshi Motome $\odot,{ }^{4}$ and Masayuki Hagiwara $\odot^{2}$ \\ ${ }^{1}$ Molecular Photoscience Research Center, Kobe University, Kobe, Hyogo 657-8051, Japan \\ ${ }^{2}$ Center for Advanced High Magnetic Field Science, Graduate School of Science, Osaka University, Toyonaka, Osaka 560-0043, Japan \\ ${ }^{3}$ Department of Advanced Materials Science, The University of Tokyo, Kashiwa, Chiba 277-8561, Japan \\ ${ }^{4}$ Department of Applied Physics, The University of Tokyo, Hongo, 7-3-1 Bunkyo, Tokyo 113-8656, Japan
}

(Received 17 September 2021; accepted 30 November 2021; published 17 December 2021)

\begin{abstract}
It is known that magnetic excitations in magnetoelectric multiferroics can be induced by the oscillation of electric polarization, called electromagnons, which often cause nonreciprocal optical phenomena. Energy diagrams of the excitations in the magnetoelectric multiferroic $\mathrm{Pb}(\mathrm{TiO}) \mathrm{Cu}_{4}\left(\mathrm{PO}_{4}\right)_{4}$ were obtained in wide ranges of magnetic fields up to $50 \mathrm{~T}$ and frequencies below $2 \mathrm{THz}$. Some of the observed resonance modes were reproduced qualitatively by a numerical analysis based on the generalized spin-wave theory. Moreover, we found that electron spin resonance (ESR) signals on some of the modes can be modulated by applying an electric field. This result implies the presence of nonreciprocal linear dichroism on the ESR signals in $\mathrm{Pb}(\mathrm{TiO}) \mathrm{Cu}_{4}\left(\mathrm{PO}_{4}\right)_{4}$.
\end{abstract}

DOI: 10.1103/PhysRevResearch.3.L042043

The magnetoelectric (ME) effects in multiferroic materials have attracted much attention in light of the potential application of such effects to electric field-controlled spin devices [1,2]. It is also noteworthy that broken symmetry, which is the origin of the ME effects, induces nonreciprocal responses [3]. The ME effects of the correlation between spin and electric polarization also affect the magnetic excitations. Electromagnons, magnetic excitations correlated with electric polarization, were observed in some multiferroic materials. Because of the electromagnons' excitation energy in the $\mathrm{GHz}$ and $\mathrm{THz}$ regions, electron spin resonance (ESR) and $\mathrm{THz}$ time-domain spectroscopy have been used to observe electromagnons. ESR is one of the most powerful measurement techniques that can provide useful microscopic information in magnetic materials. Electromagnons can be excited not only by the oscillating magnetic field components of electromagnetic waves but also by the oscillating electric fields, and thus they are of great importance for applications such as the high-speed manipulation of spins by oscillating electric fields. In addition, forbidden transitions in conventional ESR are allowed as a result of the ME effect. For example, the spin quadrupole excitation observed in $\mathrm{Sr}_{2} \mathrm{CoGe}_{2} \mathrm{O}_{7}$ [4], which cannot be excited by the oscillating magnetic field, is allowed only by the oscillating electric field. The extension of the linear ME effect to electromagnetic waves is called the optical magnetoelectric (OME) effect. The OME effect also makes it possible for nonreciprocal behaviors

\footnotetext{
*akaki@crystal.kobe-u.ac.jp

Published by the American Physical Society under the terms of the Creative Commons Attribution 4.0 International license. Further distribution of this work must maintain attribution to the author(s) and the published article's title, journal citation, and DOI.
}

such as directional dichroism and linear dichroism to occur, representing the absorption intensity change by the incident electromagnetic wave direction. Therefore, directional dichroism in multiferroic materials has been observed in optical absorption measurements at various frequencies [5-9]. The directional dichroism of electromagnon absorption has also been reported [10-14]. Nonreciprocal linear dichroism for visible light is observed in $\mathrm{Pb}(\mathrm{TiO}) \mathrm{Cu}_{4}\left(\mathrm{PO}_{4}\right)_{4}$ ( $\mathrm{PbTCPO}$ ), and magnetoelectric quadrupole domain imaging has been realized [15]. Moreover, since PbTCPO is a magnetic clustered system with square cupolas, anomalous magnetic excitations reflecting the clustering nature can be observed in ESR. In this Letter, we investigate the microscopic ME properties of $\mathrm{PbTCPO}$ experimentally and theoretically by using high-field ESR, and aim to observe the nonreciprocal behavior of magnetic excitations.

In PbTCPO (tetragonal, $P 42_{1} 2$ ), four corner-sharing $\mathrm{CuO}_{4}$ plaquettes form a magnetic square-cupola cluster $\mathrm{Cu}_{4} \mathrm{O}_{12}$, as shown in Figs. 1(a) and 1(b). These clusters possess twodimensional staggered upward and downward arrays, and thus $\mathrm{PbTCPO}$ is viewed as a square-cupola-based antiferromagnet. Below $T_{\mathrm{N}}=7 \mathrm{~K}$ at $0 \mathrm{~T}$, PbTCPO exhibits an antiferromagnetic phase transition into a low-field phase (LF), which is accompanied by the ME effects. In LF, the $a b$-plane spin components in the square-cupola cluster are regarded as a magnetic quadrupole moment that is the source of the linear ME effect. The magnetic point group is $4^{\prime} 22^{\prime}$ and allows finite components of the linear ME tensor susceptibility, $\alpha_{x x}=-\alpha_{y y}$, where $x \|$ [100] and $y \|$ [010]. Due to this linear

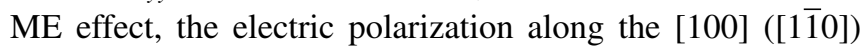
direction is linearly induced by the magnetic field applied along the [100] ([110]) direction [16]. The magnetic point group $4^{\prime} 22^{\prime}$ also allows the OME effect characterized by dynamical ME susceptibilities $\alpha_{x x}^{\omega}=-\alpha_{y y}^{\omega}$ which are responsible for the nonreciprocal linear dichroism observed in the 
(a) Q1

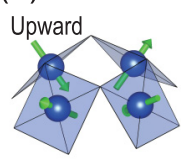

(b) Q2

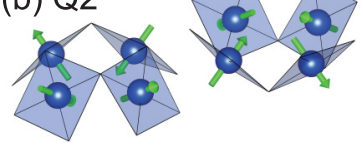

(c)

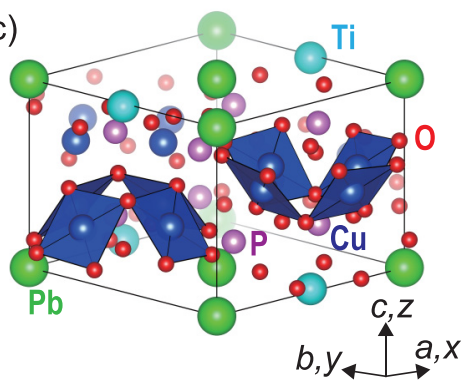

FIG. 1. Schematic of (a), (b) crystal and magnetic structures of $\mathrm{Cu}_{4} \mathrm{O}_{12}$ square cupolas and (c) crystal structure of PbTCPO. Green arrows represent $\mathrm{Cu}^{2+}$ spins in (a) Q1 and (b) Q2 domains. Solid lines in (c) represent a unit cell.

visible range [15]. The magnetization process of $\mathrm{PbTCPO}$ was investigated both experimentally and theoretically $[17,18]$. Magnetization curves for the magnetic fields applied along the [100], [110], and [001] axes were measured by a pulsed field technique [17]. For all field directions, the material shows a metamagnetic transition into a high-field phase (FI) and subsequently a transition into a saturated state. The metamagnetic transition field and saturated field are $B_{[001]}^{c 1}=12.3 \mathrm{~T}$ and $B_{[001]}^{c 2}=43.4 \mathrm{~T}$ for [001], respectively, $B_{[100]}^{c 1}=14.8 \mathrm{~T}$ and $B_{[100]}^{c 2}=47.2 \mathrm{~T}$ for [100], and $B_{[110]}^{c 1}=16.4 \mathrm{~T}$ and $B_{[110]}^{c 2}=$ $45 \mathrm{~T}$ for [110]. These magnetization processes were well reproduced by the numerical calculations [17].

The single crystals of PbTCPO used in this study were grown by the slow-cooling method [16]. The crystal orientation was determined by the Laue x-ray method. The crystal structures displayed in this Letter were drawn using VESTA software [19]. Low-field ESR spectra up to $14 \mathrm{~T}$ at $1.6 \mathrm{~K}$ and for frequencies below $0.5 \mathrm{THz}$ were taken using a homemade transmission-type ESR cryostat inserted in a 14-T superconducting magnet. High-field ESR measurements at $1.4 \mathrm{~K}$ in pulsed magnetic fields of up to $50 \mathrm{~T}$ were conducted by utilizing a far-infrared laser, Gunn oscillators, and their doublers to generate $\mathrm{THz}$ and sub- $\mathrm{THz}$ waves. An InSb hot-electron bolometer was used as a detector.

Figure 2 shows the ESR absorption spectra of PbTCPO at $1.4 \mathrm{~K}$ for magnetic fields applied along [100] [Fig. 2(a)] and [001] [Fig. 2(b)]. Since the PbTCPO sample used in the experiment has a thin plate shape with the (001) plane, the measurements in $B \|[001]$ were conducted in the Faraday configuration where the directions of the incident electromagnetic wave and the applied external magnetic field are parallel, and the measurements in $B \|[100]$ were conducted in the Voigt configuration where they are perpendicular. Between 0.8 and $1.1 \mathrm{THz}$, no resonance absorption was observed regardless of the magnetic field directions owing to the phonon absorption of the sample, which makes the transmitted light very weak. For $B \|[100]$, a baseline shift, which is probably caused by a change in dielectric permittivity, is observed at $B_{[100]}^{c 1}$.

In Fig. 3, the frequency versus resonance-field plots at 1.4-1.6 K are displayed. The solid lines are guides for the reader's eye. The gray shaded area is the phonon absorption band as mentioned above. Four zero-field energy gaps are identified: $\Delta_{1}=0.2 \mathrm{THz}, \Delta_{2}=0.6 \mathrm{THz}, \Delta_{3}=0.8 \mathrm{THz}$, and

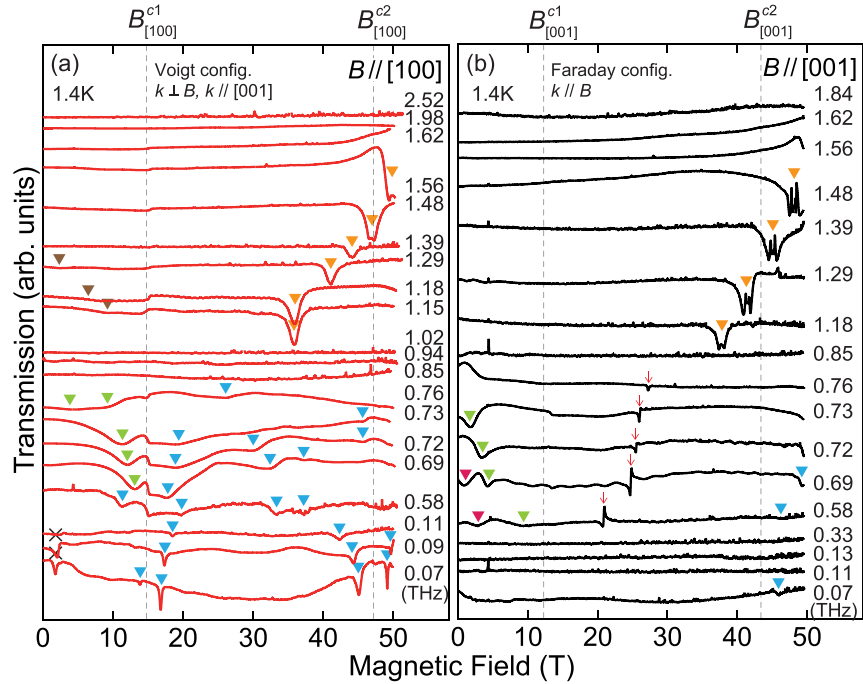

FIG. 2. Pulsed high-field ESR absorption spectra of PbTCPO at $1.4 \mathrm{~K}$ for (a) $B \|[100]$ and (b) $B \|[001]$. Vertical dashed lines indicate the metamagnetic transition fields. Arrows denote the ESR signals of DPPH (2,2-diphenyl-1-picrylhydrazyl, ESR standard marker with $g=2.0036$ ) for the field calibration. Crosses at low fields denote artifact signals originating from the cryostat. Inverse triangles show the resonance fields of the main ESR signals of $\mathrm{PbTCPO}$

$\Delta_{4}=1.35 \mathrm{THz}$. The lowest-energy branch from $\Delta_{1}$ in the zero field shows the Zeeman split in a finite magnetic field, and these upper and lower branches possess different slopes and curvatures depending on the field direction. The ESR signal on the upper branch of the lowest mode for $B \|[100]$ $\left[A_{\text {res }}\right.$ in Fig. 3(a)] is used for nonreciprocal linear dichroism measurements. We have successfully measured the nonreciprocal linear dichroism of the $A_{\text {res }}$ signal and will describe the results later.

To investigate the field-dependent ESR spectra (Figs. 2 and 3), we evaluate the imaginary part of the dynamical spin correlator

$$
\operatorname{Im} \chi^{x x}(\omega)+\operatorname{Im} \chi^{y y}(\omega)
$$

where

$$
\chi^{\mu \mu}(\omega)=i \int_{0}^{\infty} e^{i \omega t-\eta t}\left\langle\left[S^{\mu}(t), S^{\mu}(0)\right]\right\rangle d t,
$$

and

$$
S^{\mu}=\frac{1}{\sqrt{N}} \sum_{\ell} S_{\ell}^{\mu},
$$

that corresponds to the ESR absorption spectrum by the generalized spin-wave theory [20] (also known as the flavor wave theory). We take $\hbar$ unity. This method is compatible with the cluster mean-field approach [21], which has been successfully explained by the magnetoelectric behavior in the series of compounds $A \mathrm{TCPO}(A=\mathrm{Ba}, \mathrm{Sr}$, and $\mathrm{Pb})[17,21,22]$. This Letter uses the effective spin model [21] with the same parameter set used for PbTCPO in Ref. [17]. The effective spin model is described in the Supplemental Material [23]. In the cluster mean-field approach, intracupola interactions 


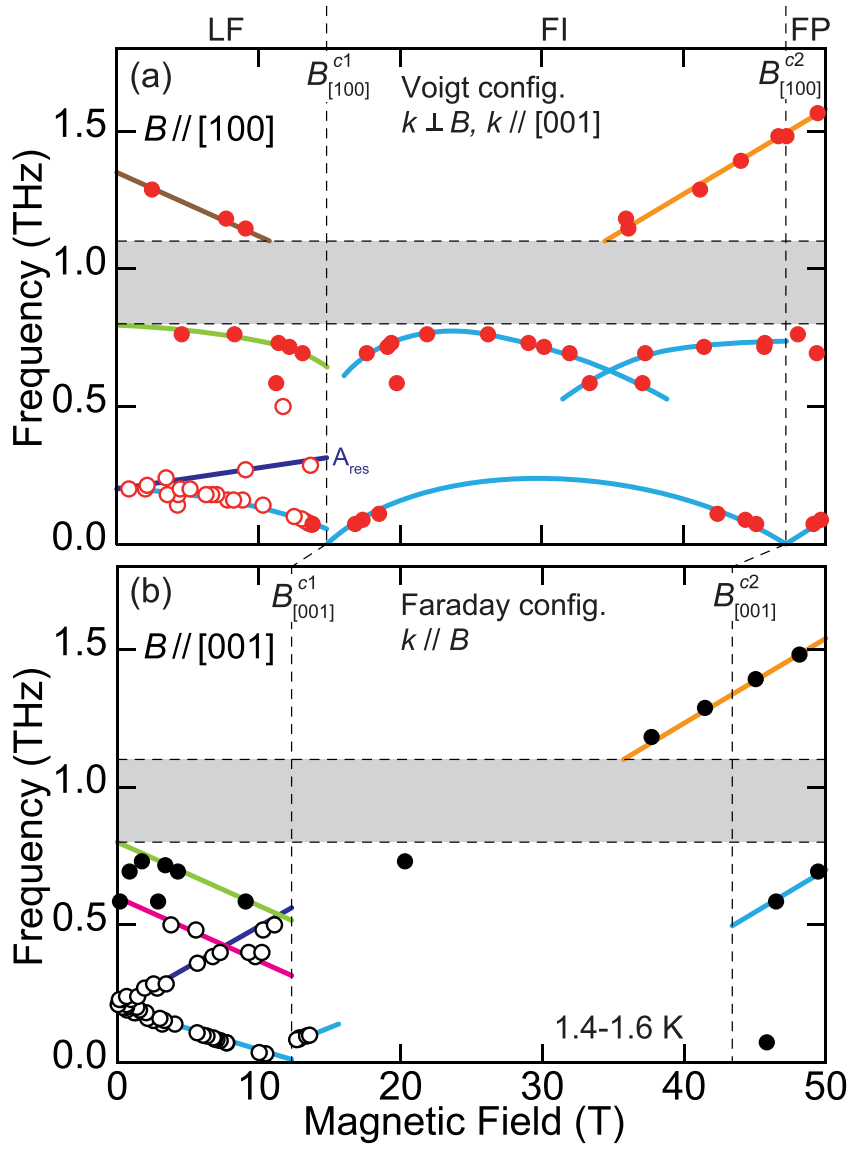

FIG. 3. Frequency-magnetic field plots of the ESR resonance fields of PbTCPO for magnetic fields parallel to the (a) [100] and (b) [001] directions. Open circles are obtained from the staticfield measurements at $1.6 \mathrm{~K}$, and solid circles are obtained from the pulsed-field measurements at $1.4 \mathrm{~K}$. The vertical dashed lines indicate the transition fields observed in the magnetization measurements. The gray shaded area represents an ESR-invisible region caused by phonon absorption. The solid lines are guides for the eye.

are treated by exact diagonalization, while intercupola interactions are treated by the mean-field approximation. The details for the calculation procedure were described in the previous report [21]. Since each cupola consisting of four $\mathrm{Cu}^{2+}$ hosting $S=1 / 2$ degrees of freedom is regarded as a cluster, the dimension of the diagonalized matrix is $2^{4}$. Leaving only bilinear contributions in terms of the boson operators after rewriting the spin Hamiltonian in the Schwinger boson representation, we obtain a one-body spin-wave Hamiltonian as in the standard linear spin-wave theory. By the Bogoliubov transformation [24] after the Fourier transformation, the spinwave Hamiltonian is diagonalized as

$$
\mathcal{H}_{\mathrm{GSW}}=\sum_{\mathbf{K}} \sum_{\ell} \omega_{\mathbf{K} \ell} \gamma_{\mathbf{K} \ell}^{\dagger} \gamma_{\mathbf{K} \ell},
$$

where $\mathbf{K}$ represents the wave number, and $\gamma_{\mathbf{K} \ell}\left(\gamma_{\mathbf{K} \ell}^{\dagger}\right)$ is the annihilator (generator) of the $\ell$ th mode with the excitation energy $\omega_{\mathbf{K} \ell}$. The dynamical spin correlation function is computed by

$$
\operatorname{Im} \chi^{\mu \mu}(\omega)=\sum_{\ell}\left|\left\langle\emptyset\left|\gamma_{\mathbf{0} \ell} S^{\mu}\right| \emptyset\right\rangle\right|^{2} \frac{\eta}{\left(\omega-\omega_{\mathbf{0} \ell}\right)^{2}+\eta^{2}},
$$
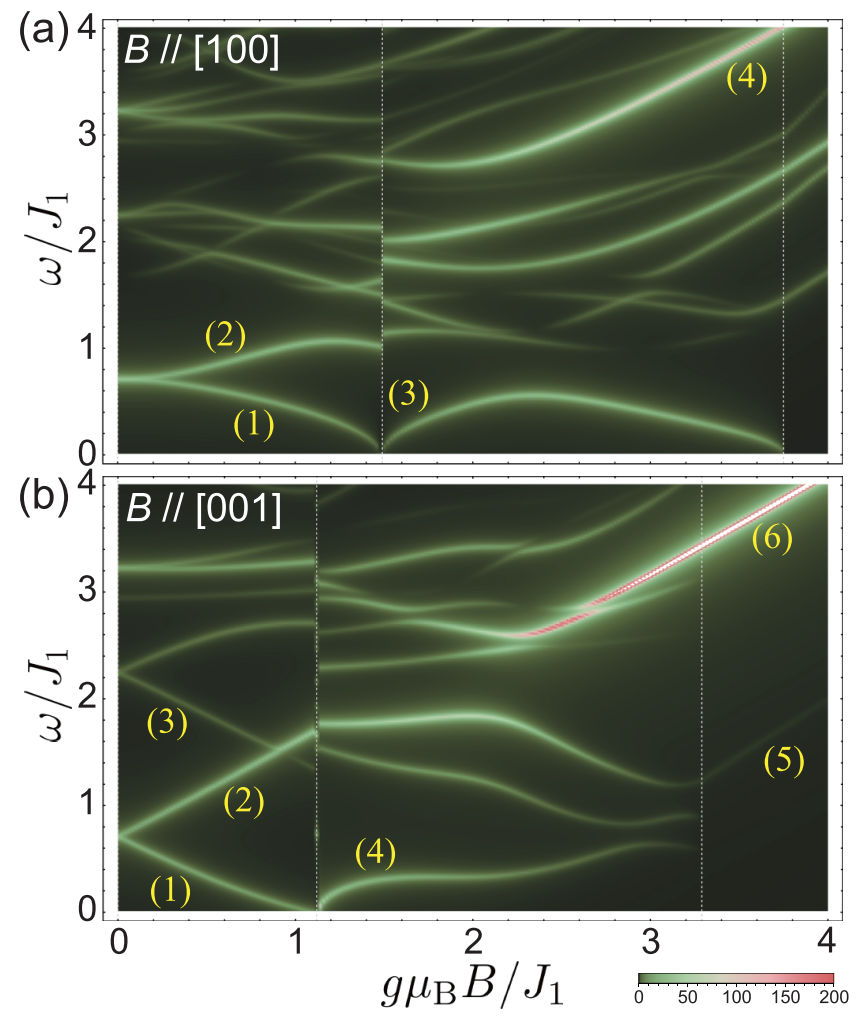

FIG. 4. ESR absorption spectra $-\left[\operatorname{Im} \chi^{x x}(\omega)+\operatorname{Im} \chi^{y y}(\omega)\right]$ computed by the generalized spin-wave theory based on the cluster mean-field approach using the effective spin Hamiltonian [21] for (a) $B \|[100]$ and (b) $B \|[001]$. The labels (1), (2), . in each panel indicate the modes corresponding to the experimentally observed ones (Fig. 3).

where $|\emptyset\rangle$ represents the vacuum, and $\eta$ is a positive infinitesimal (we set $\eta=0.02$ in the calculations). Figure 4 shows the results for $B \|[100]$ and $B \|[001]$. We find good agreement in the $B$ dependence of the modes marked as (1), (2), ... in each panel with the experimentally observed modes in Fig. 3. In the cases of both $B \|[100]$ and $B \|$ [001], we find that the lowest-energy excitation mode is split into two by applying a magnetic field. The nature of each mode, however, is quite different depending on the direction of the magnetic field. For $B \|[100]$, the lower-energy mode (1) and the next one (2) are the precession modes perpendicular and parallel to $B$, respectively. Meanwhile, for $B \|[001]$, the lower (1) and higher (2) are the anticlockwise and clockwise precession modes, respectively. Animations of these modes are shown in the Supplemental Material [23].

As our theoretical treatment is based on the spin-only model, it cannot deal with electromagnon excitations directly. However, the spin and the electric polarization are tightly coupled in multiferroic materials, hence we expect that the excitation modes found in the current analysis comprise the electromagnon excitations. If this is the case, the system exhibits nonreciprocal phenomena due to the OME effect through the electromagnon excitations. In PbTCPO, the OME effect associated with the ME susceptibilities $\alpha_{x x}^{\omega}=$ $-\alpha_{y y}^{\omega}$ induces an optical anisotropy in the (001) plane and differentiates refractive indices along the [110] and [1 $\overline{1} 0]$ 
directions [15]. Accordingly, linear dichroism, i.e., the lightpolarization-dependent difference in absorption, shows up for light propagating along the [001] direction. Moreover, the sign of the linear dichroism is reversed by switching the light propagation direction; hence, this phenomenon is called nonreciprocal linear dichroism. Furthermore, the sign of the linear dichroism is also reversed between a pair of magnetic quadrupole domains Q1 and Q2 which are mutually converted by a time-reversal operation. Consequently, the nonreciprocal linear dichroism in PbTCPO can be observed as the difference in absorption spectra between the quadrupole domains. Since the quadrupole domain can be controlled by an electric field in the presence of a magnetic field through the linear ME effect, we attempted to observe the nonreciprocal linear dichroism of magnetic excitation by an external ac electric field.

In the ESR measurements using a transmission-type probe in steady magnetic fields, thermal fluctuations cause instability in the baseline of the transmitted light intensity, which makes it difficult to compare absorption intensities. Therefore, the observation of dichroism in ESR is difficult except in cases where the effect is particularly large, as in $\mathrm{TlCuCl}_{3}$ [25]. In this study, the nonreciprocal linear dichroism was observed by measuring the ESR signal by applying an external ac electric field of $0.15 \mathrm{~Hz}$. Figure 5(a) represents the ESR absorption spectra measured by applying an ac electric field. The experimental setup is indicated in the inset of Fig. 5(b). The external magnetic field $B$ is applied parallel to the [100] direction, and using silver paste electrodes, the electric field $E$ is also applied along the same direction as $B$. The electromagnetic waves are transmitted from the back to the front side perpendicular to the face of the paper. The electromagnetic waves are polarized by a $\mathrm{Cu}$ wire grid polarizer just in front of the sample. The polarization conditions are $H^{\omega} \|$ [110] and $E^{\omega} \|[1 \overline{1} 0]$. At $286 \mathrm{GHz}$, the absorption of the $A_{\text {res }}$ mode appears around $14 \mathrm{~T}$. The ESR signal with the electric field $\pm 429 \mathrm{kV} / \mathrm{m}$ shows the rectangular modulation on the signal by the ac electric field. In this way, we successfully observed nonreciprocal linear dichroism by the reversal of the electric polarization. This method would be powerful for the study of substances with a small dichroism in ESR. The magnitude of this nonreciprocal linear dichroism is about $9 \%$ of the absorption intensity of the signal $A_{\text {res }}$. Figures 5(b) and 5(c) indicate enlarged views near $12 \mathrm{~T}$ in Fig. 5(a) with the applied ac electric field $E$ of $\pm 429 \mathrm{kV} / \mathrm{m}$. In LF with $B \|[100]$, PbTCPO has electric polarization along the [100] axis [18], and this polarization can be reversed sufficiently by $E= \pm 429 \mathrm{kV} / \mathrm{m}$. This $P-E$ hysteresis curve is shown schematically in the inset of Fig. 5(c). From the electric polarization flip due to the applied electric field, four states appear in this experiment: $1(P>0, E>0)$, 2( $P>0, E=0), 3(P<0, E<0)$, and $4(P<0, E=0)$. A comparison of the transmission among the four states reveals that the absorption is larger when the direction of the electric polarization and the direction of the magnetization (external magnetic field) are the same, and smaller when they are antiparallel. The nonreciprocal linear dichroism observed on the $A_{\text {res }}$ mode indicates that this mode is an electromagnon mode in which electric dipole excitations are allowed. Since our theoretical calculations are only for magnetic excitations and not for oscillating electric field excitations, we cannot clarify the origin of electric dipole transitions. However, our calculations
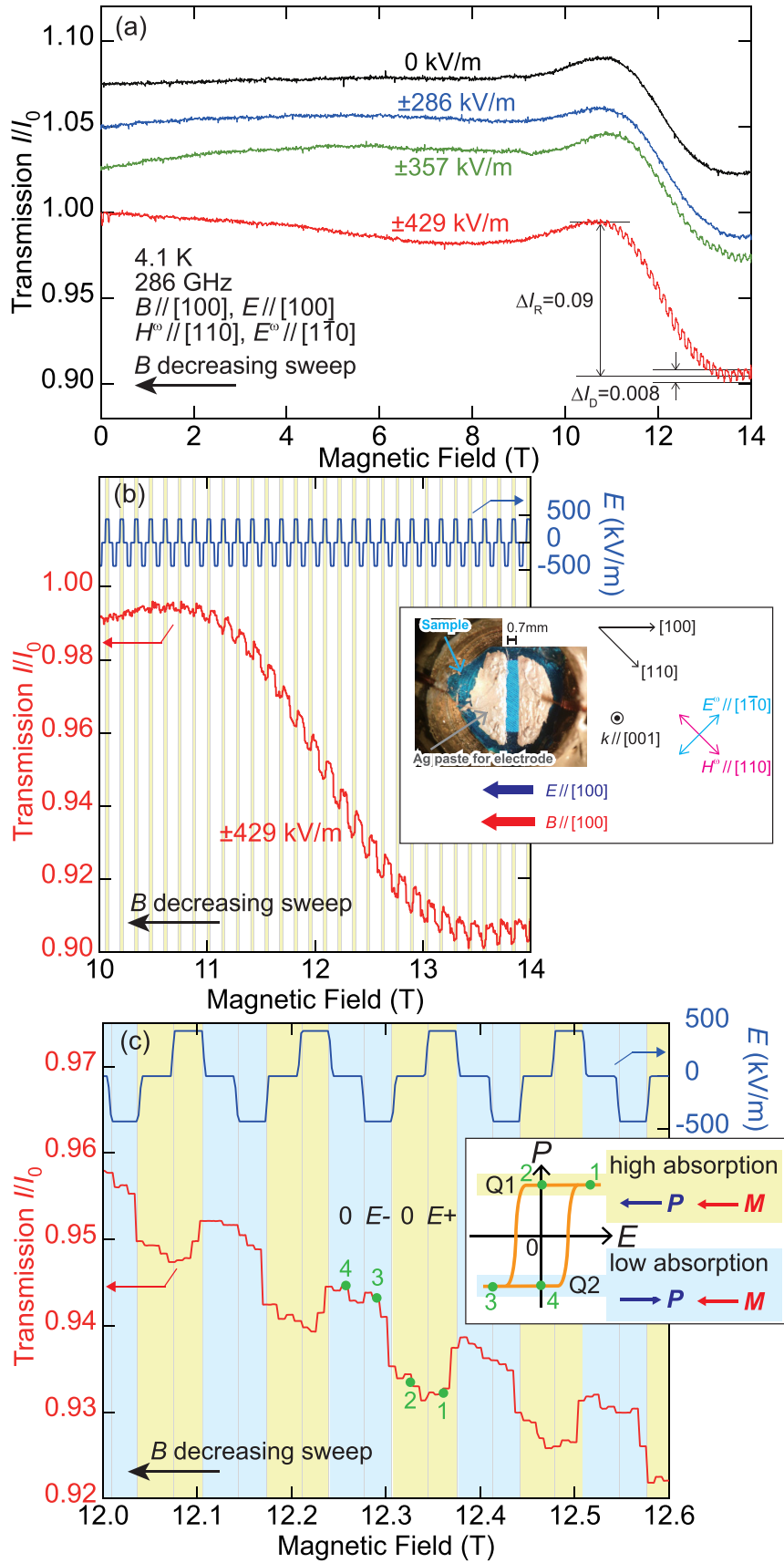

FIG. 5. (a) ESR absorption spectra in ac electric fields at $4.1 \mathrm{~K}$ for $B\|E\|[100] . I_{0}$ is transmitted light intensity at zero magnetic field. The spectra are offset by 0.025 for clarity. (b), (c) Applied electric fields and enlarged views of the ESR absorption signal for $E= \pm 429 \mathrm{kV} / \mathrm{m}$. The inset of (b) shows a photograph of the sample used in this measurement and in the experimental setup. The inset of (c) shows the schematic diagram of the $P-E$ hysteresis curve.

revealed the spin motion of $A_{\text {res }}$ as shown in the Supplemental Material (B100_1.0_(2).gif) [23]. Therefore, further development of theoretical calculations by considering electric dipole excitations will lead to the microscopic mechanism of the observed nonreciprocal phenomena. This will be a subject for future study.

In conclusion, we studied the magnetic excitations of $\mathrm{PbTCPO}$ in wide ranges of magnetic fields of up to $50 \mathrm{~T}$ 
and frequencies of up to $2.5 \mathrm{THz}$ by pulsed high-field ESR. Although phonon absorption prevented the observation of ESR signals in some regions, many resonance modes were observed. The field dependence of major resonance modes observed in the experiment was qualitatively reproduced by a theoretical analysis using the generalized spin-wave theory for an effective spin model. Furthermore, we developed an experimental approach to measure the modulation of ESR transmission signals by the alternating electric field, which enables us to uncover the nonre- ciprocal character of magnetic excitations in multiferroic materials.

This work was supported by JSPS KAKENHI Grants No. JP19K03745, No. JP19H01847, No. JP19H05823, No. JP17H06137, and No. JP17K18758. This work was carried out at the Center for Advanced High Magnetic Field Science in Osaka University under the Visiting Researcher's Program of the Institute for Solid State Physics, the University of Tokyo.
[1] T. Kimura, T. Goto, H. Shintani, K. Ishizaka, T. Arima, and Y. Tokura, Magnetic control of ferroelectric polarization, Nature (London) 426, 55 (2003).

[2] Y. Tokura, S. Seki, and N. Nagaosa, Multiferroics of spin origin, Rep. Prog. Phys. 77, 076501 (2014).

[3] Y. Tokura and N. Nagaosa, Nonreciprocal responses from noncentrosymmetric quantum materials, Nat. Commun. 9, 3740 (2018).

[4] M. Akaki, D. Yoshizawa, A. Okutani, T. Kida, J. Romhányi, K. Penc, and M. Hagiwara, Direct observation of spin-quadrupolar excitations in $\mathrm{Sr}_{2} \mathrm{CoGe}_{2} \mathrm{O}_{7}$ by high-field electron spin resonance, Phys. Rev. B 96, 214406 (2017).

[5] J. J. Hopfield and D. G. Thomas, Photon Momentum Effects in the Magneto-Optics of Excitons, Phys. Rev. Lett. 4, 357 (1960).

[6] G. L. J. A. Rikken, C. Strohm, and P. Wyder, Observation of Magnetoelectric Directional Anisotropy, Phys. Rev. Lett. 89, 133005 (2002).

[7] M. Kubota, T. Arima, Y. Kaneko, J. P. He, X. Z. Yu, and Y. Tokura, X-Ray Directional Dichroism of a Polar Ferrimagnet, Phys. Rev. Lett. 92, 137401 (2004).

[8] J. H. Jung, M. Matsubara, T. Arima, J. P. He, Y. Kaneko, and Y. Tokura, Optical Magnetoelectric Effect in the Polar $\mathrm{GaFeO}_{3}$ Ferrimagnet, Phys. Rev. Lett. 93, 037403 (2004).

[9] M. Saito, K. Taniguchi, and T. Arima, Gigantic optical magnetoelectric effect in $\mathrm{CuB}_{2} \mathrm{O}_{4}$, J. Phys. Soc. Jpn. 77, 013705 (2008).

[10] I. Kézsmárki, N. Kida, H. Murakawa, S. Bordács, Y. Onose, and Y. Tokura, Enhanced Directional Dichroism of Terahertz Light in Resonance with Magnetic Excitations of the Multiferroic $\mathrm{Ba}_{2} \mathrm{CoGe}_{2} \mathrm{O}_{7}$ Oxide Compound, Phys. Rev. Lett. 106, 057403 (2011).

[11] Y. Takahashi, R. Shimano, Y. Kaneko, H. Murakawa, and Y. Tokura, Magnetoelectric resonance with electromagnons in a perovskite helimagnet, Nat. Phys. 8, 121 (2012).

[12] S. Bordács, I. Kézsmárki, D. Szaller, L. Demkó, N. Kida, H. Murakawa, Y. Onose, R. Shimano, T. Rõõm, U. Nagel, S. Miyahara, N. Furukawa, and Y. Tokura, Chirality of matter shows up via spin excitations, Nat. Phys. 8, 734 (2012).

[13] Y. Takahashi, Y. Yamasaki, and Y. Tokura, Terahertz Magnetoelectric Resonance Enhanced by Mutual Coupling of Electromagnons, Phys. Rev. Lett. 111, 037204 (2013).

[14] Y. Okamura, F. Kagawa, M. Mochizuki, M. Kubota, S. Seki, S. Ishiwata, M. Kawasaki, Y. Onose, and Y. Tokura, Microwave magnetoelectric effect via skyrmion resonance modes in a helimagnetic multiferroic, Nat. Commun. 4, 2391 (2013).

[15] K. Kimura, T. Katsuyoshi, Y. Sawada, S. Kimura, and T. Kimura, Imaging switchable magnetoelectric quadrupole domains via nonreciprocal linear dichroism, Commun. Mater. 1, 39 (2020).

[16] K. Kimura, M. Toyoda, P. Babkevich, K. Yamauchi, M. Sera, V. Nassif, H. M. Rønnow, and T. Kimura, $A$-cation control of magnetoelectric quadrupole order in $A(\mathrm{TiO}) \mathrm{Cu}_{4}(\mathrm{PO} 4)_{4}(A=\mathrm{Ba}, \mathrm{Sr}$ and Pb), Phys. Rev. B 97, 134418 (2018).

[17] K. Kimura, Y. Kato, K. Yamauchi, A. Miyake, M. Tokunaga, A. Matsuo, K. Kindo, M. Akaki, M. Hagiwara, S. Kimura, M. Toyoda, Y. Motome, and T. Kimura, Magnetic structural unit with convex geometry: A building block hosting an exchangestriction-driven magnetoelectric coupling, Phys. Rev. Materials 2, 104415 (2018).

[18] K. Kimura, S. Kimura, and T. Kimura, Magnetoelectric Behaviors in Magnetic-Field-Induced Phases of $\mathrm{Pb}(\mathrm{TiO}) \mathrm{Cu}_{4}\left(\mathrm{PO}_{4}\right)_{4}$, J. Phys. Soc. Jpn. 88, 093707 (2019).

[19] K. Momma and F. Izumi, vESTA 3 for three-dimensional visualization of crystal, volumetric and morphology data, J. Appl. Crystallogr. 44, 1272 (2011).

[20] R. A. Muniz, Y. Kato, and C. D. Batista, Generalized spin-wave theory: Application to the bilinear-biquadratic model, Prog. Theor. Exp. Phys. 2014, 083 I01 (2014).

[21] Y. Kato, K. Kimura, A. Miyake, M. Tokunaga, A. Matsuo, K. Kindo, M. Akaki, M. Hagiwara, M. Sera, T. Kimura, and Y. Motome, Magnetoelectric Behavior from $S=1 / 2$ Asymmetric Square Cupolas, Phys. Rev. Lett. 118, 107601 (2017).

[22] Y. Kato, K. Kimura, A. Miyake, M. Tokunaga, A. Matsuo, K. Kindo, M. Akaki, M. Hagiwara, S. Kimura, T. Kimura, and Y. Motome, Magnetoelectric behavior from cluster multipoles in square cupolas: Study of $\mathrm{Sr}(\mathrm{TiO}) \mathrm{Cu}_{4}\left(\mathrm{PO}_{4}\right)_{4}$ in comparison with $\mathrm{Ba}$ and $\mathrm{Pb}$ isostructurals, Phys. Rev. B 99, 024415 (2019).

[23] See Supplemental Material at http://link.aps.org/supplemental/ 10.1103/PhysRevResearch.3.L042043 for the details of the calculation model and animations of the spin motions.

[24] J. H. P. Colpa, Diagonalization of the quadratic boson Hamiltonian, Physica A 93, 327 (1978).

[25] S. Kimura, M. Matsumoto, and H. Tanaka, Electrical Switching of the Nonreciprocal Directional Microwave Response in a Triplon Bose-Einstein Condensate, Phys. Rev. Lett. 124, 217401 (2020). 